\section{Kompass \\ Pneumologie}

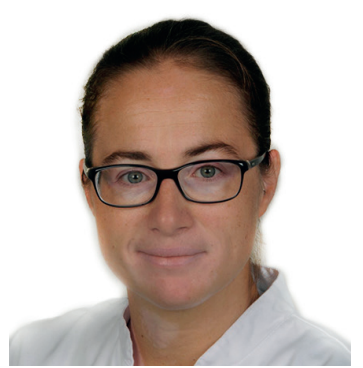

\section{Eva Lücke}

Universitätsklinikum und Medizinische Fakultät der Otto-von-Guericke-Universität, Klinik für Pneumologie, Magdeburg, Deutschland

\title{
Eva Lücke
}

\section{Trägerin des «Förderpreises der Mitteldeutschen Gesellschaft für Pneumologie und Thoraxchirurgie (MDGP)» 2020}

\section{Forschungsschwerpunkte}

- obstruktive Atemwegserkrankung

- Asthma bronchiale

- kardiorespiratorische Interaktion

\section{Akademischer Werdegang}

• 2004-2010: Studium der Humanmedizin an der Otto-von-Guericke-Universität Magdeburg

- 2010-2018: Facharztweiterbildung zum Facharzt für Innere Medizin und Pneumologie, Klinik für Pneumologie, Universitätsklinik Magdeburg und Promotion zum Dr. med.

• seit 2018: Oberärztin in der Klinik für Pneumologie, Universitätsklinik Magdeburg

\section{Homepage}

- http://kpne.med.ovgu.de

Wie kam es dazu, dass Sie sich in Ihren nun ausgezeichneten Forschungsarbeiten mit dem Einsatz von Inhalatoren bei der Therapie von Patientinnen und Patienten mit chronisch-obstruktiven Atemwegserkrankungen beschäftigen?

In der täglichen klinischen Arbeit mit den Patientinnen und Patienten fällt immer wieder auf, dass viele den verordneten Inhalator nicht korrekt anwenden und somit die erwartete Wirksamkeit nicht gegeben ist. Für den Erfolg der Therapie ist die korrekte Anwendung eines Inhalationsdevices jedoch essentiell. Bei manchen Patienten und Patienten besteht hoher Schulungsbedarf, andere wiederum können auch nach der intensiven Beübung manche Devices nicht korrekt nutzen, so dass wir wechseln müssen. Es existierten keine industrieunabhängigen Studien zu dem Thema.

\section{Was sind die zentralen Erkenntnisse Ihrer Forschungen?}

Wir haben die Präferenzen für häufig verwendete Inhalationsgeräte in Deutschland bei Patienten mit chronisch-obstruktiven Atemwegserkrankungen untersucht. Wir sind außerdem der Frage nachgegangen, welche Eigenschaften eines Inhalationsgerätes für die Anwenderin/den Anwender besonders wichtig sind und welche Auswirkungen Alter, Ge- 
schlecht, die Art der Erkrankungen (Asthma bronchiale oder COPD), sowie kognitive und motorische Einschränkungen auf die Gerätepräferenz und Handhabungsfehler haben können. Wir haben dies auch für geriatrische Patientinnen und Patienten untersucht.

105 Patientinnen und Patienten mit Asthma (58\%) oder COPD (42\%) und 106 stationäre inhalationsnaive geriatrische Patientinnen und Patienten (Alter $\geq 70$ Jahre) haben an den beiden prospektiven, offenen Querschnittstudien teilgenommen. Um die Inhalationstechnik und Handhabungsfehler mit 10 verschiedenen Geräten in der Asthma-/COPD-Kohorte und 9 Geräten in der geriatrischen Patientenkohorte objektiv zu bewerten, wurden Checklisten verwendet. Es wurden Placebo-Devices verwendet. Die geriatrischen Patientinnen und Patienten wurden einer Untersuchung auf kognitive, motorische und feinmotorische Fähigkeiten unterzogen, bevor die Verwendung der 9 verschiedenen Inhalatoren in randomisierter Reihenfolge demonstriert wurde. Für jedes Gerät wurden alle 211 Patientinnen und Patienten gebeten, die Handhabung zu testen, die Geräteeigenschaften zu bewerten und jeweils das Gerät zu benennen, das sie am meisten oder am wenigsten bevorzugen würden.

Mit der Vergabe des Förderpreises der MDGP sollen hervorragende klinische oder experimentelle Arbeiten aufdem Gebiet der Pneumologie, der Thoraxchirurgie, der pneumologischen Allergologie und der pneumologischen Versorgungsforschung unterstützt werden. Wo zeigt sich in Ihrem Forschungsansatz und Ihren Forschungsergebnissen die Relevanz für die klinische Praxis besonders deutlich? Die Studienteilnehmerinnen und -teilnehmer wurden gebeten, die Handhabung der Devices zu testen, die Geräteeigenschaften zu bewerten und ein favorisiertes Gerät zu benennen. Wir haben durch unsere Arbeit industrieunabhängige Ergebnisse generiert, die eine Hilfestellung bei der Verordnung von inhalativen Medikamenten ermöglichen und den Herstellern Daten liefern, welche dann möglicherweise dazu dienen können, einzelne Eigenschaften zu verbessern, um so die Therapie dieser Patientinnen und Patienten zu optimieren. Die geriatrischen Patientinnen und Patienten benötigten durchschnittlich 2,47 Versuche zur fehlerfreien Anwendung, also länger als die Patienten und Patienten der Asthma-/ COPD-Kohorte (im Durchschnitt 1,22 Versuche).

Was ist aus Ihrer Erfahrung besonders wichtig für die Schnittstelle zwischen Forschung und klinischer Medizin?

Forschungsaspekte sollten schon früh im Medizinstudium ihren Stellenwert erlangen, um das Interesse der Studierenden zu wecken. Wichtig es auch, die Bedeutung der Kooperation und des regelmäBigen Austausches zwischen den verschiedenen Forschungsgruppen und klinisch tätigen Kolleginnen und Kollegen zu stärken und zu intensivieren. Bestimmte Fragestellungen können in der gemeinsamen Interaktion deutlich zielführender bearbeitet werden.
Gibt es schon neue Projekte, mit denen Sie sich in Zukunft beschäftigen werden?

Ja, mehrere.

In unseren aktuellen und zukünftigen Projekten beschäftigen wir uns mit den bidirektionalen Wechselwirkungen zwischen Infektionserregern und der bronchialen Inflammation bei obstruktiven Atemwegserkrankungen. Dies geschieht sowohl in tierexperimentellen, als auch in klinischen Projekten, wobei mein Schwerpunkt in der klinischen und patientenbezogenen Forschung liegt.

Wenn Sie sich etwas für Ihre weitere Forschung bzw. für die pneumologische Forschung insgesamt wünschen dürften, was wäre das?

Wie wir gerade in der COVID-19-Pandemie erleben, sind Erkrankungen der Atmungsorgane von zentraler Bedeutung. Wir bemerken aber auch, dass es vielfältiger Interaktionen mit anderen klinischen und theoretischen Fachrichtungen bedarf. Diese Interdisziplinarität muss weiterentwickelt werden. Und selbstverständlich müssen ausreichende Freiräume für die Forschung vorhanden sein.

Womit schaffen Sie für sich selbst und für Ihr Umfeld einen Ausgleich zu Ihrem Einsatz für die Forschung?

Reisen mit der Familie sind für mich eine sehr gute Ablenkung. In der aktuellen Zeit ist dies leider nicht möglich. Als guter Ausgleich bleibt aber auf jeden Fall das Laufen in den umliegenden Wäldern und Elbauen (allein oder mit Freunden) und das Lesen. Das geht auch in Zeiten der Corona-Pandemie.

Haben Sie ganz herzlichen Dank für das Interview, Frau Dr. Lücke!

Das Interview führte unser Redakteur Patrick Welge

\section{ANGABEN ZUM PREIS}

Die Mitteldeutsche Gesellschaft für Pneumologie und Thoraxchirurgie (MDGP) ist die jüngste und größte von vier wissenschaftlichen Regionalgesellschaften für Pneumologie in Deutschland. Die MDGP verfolgt das Ziel, alle das Fachgebiet Pneumologie und Thoraxchirurgie sowie die Berufsverbände in den Bundesländern Sachsen, Sachsen-Anhalt und Thüringen betreffenden Aufgaben zu fördern und zu unterstützen. Mit der Vergabe eines Förderpreises sollen hervorragende klinische oder experimentelle Arbeiten auf dem Gebiet der Pneumologie, der Thoraxchirurgie, der pneumologischen Allergologie und der pneumologischen Versorgungsforschung unterstützt werden.

Weitere Informationen:

https://www.mdgp.de/foerderpreis/ 